\title{
The Effect of Two Infectious Diseases and Harvesting in the Eco-Epidemiological Model
}

\author{
Rasha Majeed Yaseen \\ Department of Mechatronics, Al-Khwarizmi College of Engineering, University of Baghdad. \\ E-mail: rasha.majeed1@gmail.com.
}

\begin{abstract}
In this paper, an Eco-Epidemiological model with different infectious diseases in prey population and the optimal harvesting in predator population is proposed and studied. Linear type used to describe the functional response. Sufficient and necessary conditions for existence each equilibrium points of the system are established, the bounded and stability analysis of all possible equilibrium points are studied. The effect of harvest on the stability of this system is investigated. Finally, the dynamical behavior of system is discussed using Numerical simulation.
\end{abstract}

Keywords: SIS epidemics disease, Eco-Epidemiological model, Harvest management; stability analysis.

\section{Introduction}

Diseases in a prey-predator system have received significant interest in recent years. In fact, any given environment may contain many or hundreds of species. Since any species has at least the potential to interact with any other species in its environment, the possibility of spread of the diseases in a community rapidly becomes astronomical as the number of infected species in the environment increases. Therefore, it is more of biological significance to study the effect of disease on the dynamical behavior of interacting species. The first model described a prey-predator model involving disease in prey species formulated by Anderson and May [1], Later on many researchers, especially in the last two decades, have proposed and studied different predatorprey models in presence of disease in one of the species see for example [2-11] and the references there in. The effect of constant-rate harvesting on the dynamics of predator-prey systems has been investigated by many authors, see, for example [12-16] and the references there in. On the other hand, many researchers proposed and study eco-epidemical models containing two disease strains in the same population. see for example $[17,19]$ and the references there in. On contrast to all the above studies, in this paper a prey-predator model involving, in additional to harvest in predator species, two different SIS infectious diseases in prey species is proposed and analyzed. It is assumed that both the diseases spread within prey population by contact, between susceptible individuals and infected individuals. Furthermore, in this model, using linear functional response and linear incidence rate to describe spread both of diseases first and second.

\section{Mathematical Model}

To describe the model for an EcoEpidemiological system, we consider the following notation:

1. Let $N(t)$ and $p(t)$ be the population densities of the prey species and predator species at time $t$, respectively.

2 . The prey grows logistically with intrinsic growth rate $h_{1}>0$ and carrying capacity $h_{2}>0$.

3. There are two different SIS epidemic diseases spread among the prey population and it transmitted between the prey individuals (but not the predator) by contact, according to linear incidence rate with first and second infection rate constants $h_{3}>0$ and $h_{4}>0$, respectively. Therefore, the total prey population is divided into three classes: susceptible that is denoted by $x(t)$, infected by first disease that is denoted by $y(t)$ infected by second disease that is denoted by $z(t)$. Hence at any time $t$ the total prey population is $N(t)=x(t)+y(t)+z(t)$.

4. The predator preys upon prey according to linear functional response with maximum attack rates $h_{5}>0, h_{9}>0$ and $h_{11}>0$, 
respectively. Furthermore it is assumed that $e>0$ represent the conversion rate constant

5 . Both of the infected prey can be recovered and become susceptible again with recovery rate constant $h_{6}>0$ and $h_{7}>0$, respectively.

6. Furthermore it is assumed that there is disease induced mortality rate represented by $h_{8}>0$ and $h_{10}>0$, respectively.

7. The predator grows logistically with intrinsic growth rate $h_{12}>0$ and carrying capacity $h_{13}>0$.

8. Finally, $q>0$ is the catch ability co-efficient of the predator, $E>0$ is the harvesting effort and $q E p$ is the catch-rate function based on the CPUE (catch-per-unit-effort) hypothesis.

Consequently, the model with the above assumptions can be written in the following form:

$$
\begin{aligned}
\frac{d x}{d t} & =x\left[h_{1}\left(1-\frac{x+y+z}{h_{2}}\right)-h_{3} y-h_{4} z-h_{5} p\right] \\
& +h_{6} y+h_{7} z \\
\frac{d y}{d t} & =y\left(h_{3} x-h_{6}-h_{8}-h_{9} p\right) \\
\frac{d z}{d t} & =z\left(h_{4} x-h_{7}-h_{10}-h_{11} p\right) \\
\frac{d p}{d t} & =p\left[h_{12}\left(1-\frac{p}{h_{13}}\right)+e h_{5} x+e h_{9} y+e h_{11} z-q E\right]
\end{aligned}
$$

The system (1) has the following domain $\mathfrak{R}_{+}^{4}=\{(x, y, z, p), x \geq 0, y \geq 0, z \geq 0$ and $p \geq 0\}$.

Moreover, the above four nonlinear differential equations are continuously differentiable on int. $\mathfrak{R}_{+}^{4}$ and hence they are Lipschizian on $\mathfrak{R}_{+}^{4}$. Thus, for each set of initial conditions, say $x(0) \geq 0, y(0) \geq 0, z(0) \geq 0$ and $p(0) \geq 0$, system (1) has a unique solution. Therefore, the domain $\Re_{+}^{4}$ is an invariant for the system (1). Further in the following theorem the sufficient condition for uniformly bounded of the solution of the system (1) is established.

\section{Theorem (1):}

All the trajectories of system (1), which initiate in $\mathfrak{R}_{+}^{4}$ are uniformly bounded.

\section{Proof:}

From the first equation of system (1) we in the absence of diseases and predator obtain that $\frac{d x}{d t} \leq h_{1} x\left(1-\frac{x}{h_{2}}\right)$

From the four equation in the absence of prey we have $\frac{d p}{d t} \leq h_{12} p\left(1-\frac{p}{h_{13}}\right)$

Clearly by solving the above differential inequalities we get $\lim _{t \rightarrow \infty} \sup x(t) \leq h_{2}$ and $\lim _{t \rightarrow \infty} \sup p(t) \leq h_{13}$

Define the function $\mathrm{M}(t)=x(t)+y(t)+z(t)+\frac{1}{e} p(t)$ and then take its time derivative along the solution of system (1), gives

$$
\begin{aligned}
\frac{d \mathrm{M}}{d t} & \leq h_{1} x+p(e)^{-1}\left(h_{12}-q E\right)-\phi y-\phi z \\
& \leq \pi-\phi \mathrm{M} \text { where } \phi=\min \left\{h_{8}, h_{10}\right\}
\end{aligned}
$$$$
\text { and } \pi=\left(h_{1}+\phi\right) h_{2}+h_{13}(e)^{-1}\left(\left(h_{12}-q E\right)+\phi\right)
$$

Now, by using Gronwall lemma [20], it obtains that $0<\mathrm{M}(t) \leq \mathrm{M}(0) e^{-\phi t}+\pi(\phi)^{-1}\left(1-e^{-\phi t}\right)$ which yields $\lim _{t \rightarrow \infty} \sup \mathrm{M}(t) \leq \pi(\phi)^{-1}$ that is independent of the initial conditions.

For later purposes, it is necessary to have the Jacobian of system(1) at hand, it is reported below.

$$
J_{k}=\left(\beta_{i j}^{[k]}\right)=\left[\begin{array}{cccc}
\beta_{11}^{[k]} & \beta_{12}^{[k]} & \beta_{13}^{[k]} & \beta_{14}^{[k]} \\
\beta_{21}^{[k]} & \beta_{22}^{[k]} & 0 & \beta_{24}^{[k]} \\
\beta_{31}^{[k]} & 0 & \beta_{33}^{[k]} & \beta_{34}^{[k]} \\
\beta_{41}^{[k]} & \beta_{42}^{[k]} & \beta_{43}^{[k]} & \beta_{44}^{[k]}
\end{array}\right]
$$

Where: $i=1,2,3,4 ; j=1,2,3,4 ; k=0,1, \ldots, 9$ and

$$
\begin{aligned}
& \beta_{11}^{[k]}=h_{1}\left(1-\frac{2 x}{h_{2}}\right)-\left(h_{3}+\frac{h_{1}}{h_{2}}\right) y-\left(h_{4}+\frac{h_{1}}{h_{2}}\right) z-h_{5} p ; \\
& \beta_{12}^{[k]}=\frac{-h_{1}}{h_{2}} x-h_{3} x+h_{6} ; \beta_{13}^{[k]}=\frac{-h_{1}}{h_{2}} x-h_{4} x+h_{7} ; \\
& \beta_{14}^{[k]}=-h_{5} x ; \beta_{21}^{[k]}=h_{3} y ; \beta_{22}^{[k]}=h_{3} x-h_{6}-h_{8}-h_{9} p ; \\
& \beta_{23}^{[k]}=0 ; \beta_{24}^{[k]}=-h_{9} y ; \beta_{31}^{[k]}=h_{4} z ; \beta_{32}^{[k]}=0 ; \\
& \beta_{42}^{[k]}=e h_{9} p ; \beta_{33}^{[k]}=h_{4} x-h_{7}-h_{10}-h_{11} p ; \\
& \beta_{34}^{[k]}=-h_{11} z ; \beta_{41}^{[k]}=e h_{5} p ; \beta_{43}^{[k]}=e h_{11} p ; \\
& \beta_{44}^{[k]}=h_{12}\left(1-\frac{2 p}{h_{13}}\right)+e h_{5} x+e h_{9} y+e h_{11} z-q E
\end{aligned}
$$




\section{Equilibrium points}

System (1) has the following equilibrium:

1. The vanishing equilibrium point $E_{0}=(0,0,0,0)$ always exists.

2. The free diseases and predator and diseases equilibrium point $E_{1}=\left(x_{1}, 0,0,0\right)$ where $x_{1}=h_{2}, E_{1}$ always exists.

3. The free prey equilibrium point $E_{2}=\left(0,0,0, p_{2}\right)$ where:

$p_{2}=h_{13}\left(h_{12}\right)^{-1}\left[h_{12}-q E\right]$ exists if and only if $h_{12}>q E$.

4. The first disease equilibrium point $E_{3}=\left(x_{3}, y_{3}, 0,0\right)$ where: $x_{3}=\frac{h_{6}+h_{8}}{h_{3}}$ and $y_{3}=\frac{h_{1} x_{3}\left(h_{2}-x_{3}\right)}{\left(h_{2} h_{8}+h_{1} x_{3}\right)}$, exists uniquely in the interior of the first quadrant of $x y$-plane under the following necessary and sufficient condition $x_{3}<h_{2}$.

5. The second disease equilibrium point $E_{4}=\left(x_{4}, 0, z_{3}, 0\right)$ where:

$x_{4}=\left(h_{7}+h_{10}\right)\left(h_{4}\right)^{-1}$ and $z_{4}=\frac{h_{1} x_{4}\left(h_{2}-x_{4}\right)}{\left(h_{2} h_{10}+h_{1} x_{4}\right)}$

exists uniquely in the interior of the first quadrant of $x z$-plane under the following necessary and sufficient condition $x_{4}<h_{2}$

6. The susceptible prey-predator equilibrium point $E_{5}=\left(x_{5}, 0,0, p_{5}\right) \quad$ where: $x_{5}=\frac{h_{2}\left[h_{5} h_{13} q E+h_{1} h_{12}-h_{5} h_{12} h_{13}\right]}{\left[h_{1} h_{12}+e h_{2} h_{13}\left(h_{5}\right)^{2}\right]} \quad$ and $p_{5}=\frac{h_{1}\left(h_{2}-x_{5}\right)}{h_{2} h_{5}}$, exists uniquely in the interior of the first quadrant of $x p$-plane if and only if $x_{5}<h_{2}$ and $h_{5} h_{13} q E+h_{1} h_{12}>h_{5} h_{12} h_{13}$

7. The free first disease equilibrium point $E_{6}=\left(x_{6}, 0, z_{6}, p_{6}\right)$ where

$p_{6}=\left(h_{4} x_{6}-h_{7}-h_{10}\right)\left(h_{11}\right)^{-1}$

and

$z_{6}=\frac{x_{6}\left[h_{1}\left(h_{2}-x_{6}\right)-h_{2} h_{5} p_{6}\right]}{\left[h_{1} x_{6}+h_{2} h_{4} x_{6}-h_{2} h_{7}\right]} \quad$ while $x_{6}$

represents a positive root of the equation $A_{1} x^{2}+A_{2} x+A_{3}=0$ where:

$$
\begin{aligned}
A_{1} & =e h_{1} h_{5} h_{11} h_{13}-h_{1} h_{4} h_{12}-h_{2} h_{12}\left(h_{4}\right)^{2} \\
& -e h_{1} h_{13}\left(h_{11}\right)^{2}
\end{aligned}
$$

$$
\begin{aligned}
& A_{2}=h_{1} h_{11} h_{12} h_{13}+h_{1} h_{7} h_{12}+h_{1} h_{10} h_{12} \\
& \quad+h_{2} h_{4} h_{10} h_{12}+h_{2} h_{4} h_{11} h_{12} h_{13}+2 h_{2} h_{4} h_{7} h_{12} \\
& \quad+e h_{1} h_{2} h_{13}\left(h_{11}\right)^{2}+e h_{2} h_{5} h_{10} h_{11} h_{13} \\
& \quad-q E h_{1} h_{11} h_{13}-q E h_{2} h_{4} h_{11} h_{13} \\
& A_{3}=q E h_{2} h_{7} h_{11} h_{13}-h_{2} h_{7} h_{10} h_{12}-h_{2} h_{12}\left(h_{7}\right)^{2} \\
& \quad-h_{2} h_{7} h_{11} h_{12} h_{13}
\end{aligned}
$$

Obviously, $E_{6}$ exists uniquely in the interior of the first octant of $x z p$ - space if and only if the following conditions are hold.

$A_{1}>0 ; A_{3}<0$

or

$A_{1}<0 ; A_{3}>0$

with $h_{7}+h_{10}<h_{4} x_{6} ; h_{2} h_{5} p_{6}+h_{1} x_{6}<h_{1} h_{2}$ and $h_{2} h_{7}<h_{1} x_{6}+h_{2} h_{4} x_{6}$

8. The free second disease equilibrium point $E_{7}=\left(x_{7}, y_{7}, 0, p_{7}\right)$ where:

$y_{7}=\frac{\left[q E h_{13}-h_{12} h_{13}+h_{12} p_{7}-e h_{5} h_{13} x_{7}\right]}{e h_{9} h_{13}}$ and $p_{7}=\frac{\left(h_{3} x_{7}-h_{6}-h_{8}\right)}{h_{9}}$ while $x_{7}$ represents a positive root of the equation $B_{1} x^{2}+B_{2} x+B_{3}=0$ where:

$$
\begin{aligned}
B_{1}= & e h_{1} h_{5} h_{9} h_{13}-h_{1} h_{3} h_{12}-h_{2} h_{12}\left(h_{3}\right)^{2} \\
& -e h_{1} h_{13}\left(h_{9}\right)^{2} \\
B_{2}= & e h_{1} h_{2} h_{13}\left(h_{9}\right)^{2}+e h_{2} h_{5} h_{6} h_{9} h_{13} \\
& +e h_{2} h_{5} h_{8} h_{9} h_{13}+h_{2} h_{3} h_{6} h_{12}- \\
& e h_{2} h_{5} h_{6} h_{9} h_{13}-q E h_{2} h_{3} h_{9} h_{13} \\
& +h_{2} h_{3} h_{9} h_{12} h_{13}+h_{2} h_{3} h_{6} h_{12}+ \\
& h_{2} h_{3} h_{8} h_{12}-q E h_{1} h_{9} h_{13}+h_{1} h_{9} h_{12} h_{13} \\
& +h_{1} h_{6} h_{12}+h_{1} h_{8} h_{12} \\
B_{3}= & q E h_{2} h_{6} h_{9} h_{13}-h_{2} h_{6} h_{9} h_{12} h_{13} \\
& -h_{2} h_{12}\left(h_{6}\right)^{2}-h_{2} h_{6} h_{8} h_{12}
\end{aligned}
$$

Obviously, $E_{7}$ exists uniquely in the interior of the first octant of $x y p$ - space if and only if the one set of following conditions are hold.

$B_{1}>0 ; B_{3}<0$

or

$B_{1}<0 ; B_{3}>0$

with $h_{13}\left(h_{12}+e h_{5} x_{7}\right)<q E h_{13}+h_{12} p_{7} \quad$ and $\left(h_{6}+h_{8}\right)<h_{3} x_{7}$

9. The free predator equilibrium point

$$
\begin{aligned}
& E_{8}=\left(x_{8}, y_{8}, z_{8}, 0\right) \text { where } \\
& x_{8}=\left\{\frac{h_{6}+h_{8}}{h_{3}}, \frac{h_{7}+h_{10}}{h_{4}}\right\}
\end{aligned}
$$

and 
$y_{8}=\frac{h_{1} x_{8}\left[h_{2}-x_{8}\right]-h_{3} z_{8}\left[h_{2} h_{10}+h_{1} x_{8}\right]}{\left[h_{2} h_{8}+h_{1} x_{8}\right]}, \quad E_{8}$

exists uniquely in the interior of the first octant of $x y z$ - space under the following necessary and sufficient conditions $x_{8}<h_{2}$

$$
\frac{\left(h_{6}+h_{8}\right)}{h_{3}}=\frac{\left(h_{7}+h_{10}\right)}{h_{4}} \text { and } z_{8}<\frac{h_{1} x_{8}\left[h_{2}-x_{8}\right]}{\left[h_{2} h_{10}+h_{1} x_{8}\right]}
$$

10. The coexistence equilibrium point $E_{9}=\left(x_{9}, y_{9}, z_{9}, p_{9}\right)$ where

$x_{9}=\frac{h_{11}\left(h_{6}+h_{8}\right)-h_{9}\left(h_{7}+h_{10}\right)}{\left(h_{3} h_{11}-h_{4} h_{9}\right)}$;

$p_{9}=\left\{\frac{\left[h_{3} x_{9}-\left(h_{6}+h_{8}\right)\right]}{h_{9}}, \frac{\left[h_{4} x_{9}-\left(h_{7}+h_{10}\right)\right]}{h_{11}}\right\}$

$y_{9}=\frac{\left[h_{13}\left(q E-e h_{5} x_{9}-h_{12}-e h_{11} z_{9}\right)+h_{12} p_{9}\right]}{e h_{9} h_{13}}$

$z_{9}=\frac{\left[\begin{array}{l}e h_{9} h_{13} x_{9}\left[h_{1}\left(h_{2}-x_{9}\right)-h_{2} h_{5} p_{9}\right] \\ +\left[\begin{array}{l}q E h_{13}-e h_{5} h_{13} x_{9}-h_{12} h_{13}+h_{12} p_{9} \\ \times\left[h_{2} h_{6}-\left(h_{1}+h_{2} h_{3}\right) x_{9}\right]\end{array}\right]\end{array} e h_{13} x_{9}\left[h_{2}\left(h_{6} h_{11}-h_{7} h_{9}\right)-\left(\begin{array}{l}h_{9}\left(h_{1}+h_{2} h_{4}\right) \\ -h_{11}\left(h_{1}+h_{2} h_{3}\right)\end{array}\right)\right.\right.}{A}$

exists uniquely in the Int. $\Re_{+}^{4}$ under the following necessary and sufficient condition

$$
\begin{aligned}
& h_{4} h_{9}<h_{3} h_{11}, h_{9}\left(h_{7}+h_{10}\right)<h_{11}\left(h_{6}+h_{8}\right), \\
& e h_{5} h_{13} x_{9}+h_{12} h_{13}+e h_{11} h_{13} z_{9}<q E h_{13}+h_{12} p_{9} \\
& \max \left\{\frac{\left(h_{6}+h_{8}\right)}{h_{3}}, \frac{\left(h_{7}+h_{10}\right)}{h_{4}}\right\}<x_{9} \\
& x_{9}<\min \left\{h_{2}, \frac{h_{2} h_{6}}{\left(h_{1}+h_{2} h_{3}\right)}\right\}, p_{9}<\frac{h_{1}\left(h_{2}-x_{9}\right)}{h_{2} h_{5}}
\end{aligned}
$$

\section{Stability analysis of the system}

At the equilibrium point $E_{0}$ the eigenvalues are

$$
h_{1} ;-\left(h_{6}+h_{8}\right) ;-\left(h_{7}+h_{10}\right) ;\left(h_{12}-q E\right)
$$

showing that it is an unstable saddle.

\section{Theorem (2):}

The equilibrium point $E_{1}$ is locally asymptotically stable in $\mathfrak{R}_{+}^{4}$ if and only if $\left.\begin{array}{l}h_{2}<\min \left\{\left(h_{6}+h_{8}\right)\left(h_{3}\right)^{-1} ;\left(h_{7}+h_{10}\right)\left(h_{4}\right)^{-1}\right\} \\ \left(h_{12}+e h_{2} h_{5}\right)<q E\end{array}\right\}$

\section{Proof:}

The Jacobian matrix of the system (1) at $E_{1}$ is given by:
$J_{1}=\left(\begin{array}{cccc}\beta_{11}^{[1]} & \beta_{12}^{[1]} & \beta_{13}^{[1]} & \beta_{14}^{[1]} \\ 0 & \beta_{22}^{[1]} & 0 & 0 \\ 0 & 0 & \beta_{33}^{[1]} & 0 \\ 0 & 0 & 0 & \beta_{44}^{[1]}\end{array}\right)$ where:

$\beta_{11}^{[1]}=-h_{1}, \beta_{12}^{[1]}=\left(h_{6}-h_{1}-h_{2} h_{3}\right), \beta_{14}^{[1]}=-h_{2} h_{5}$,

$\beta_{13}^{[1]}=\left(h_{7}-h_{1}-h_{2} h_{4}\right), \beta_{22}^{[1]}=\left(h_{2} h_{3}-h_{6}-h_{8}\right)$,

$\beta_{33}^{[1]}=\left(h_{2} h_{4}-h_{7}-h_{10}\right), \beta_{44}^{[1]}=\left(h_{12}+e h_{2} h_{5}-q E\right)$,

So, the characteristic equation of $J_{1}$ can be written by

$$
\begin{aligned}
0 & =\left(-h_{1}-\lambda_{x}^{[1]}\right)\left(\left(h_{2} h_{3}-h_{6}-h_{8}\right)-\lambda_{y}^{[1]}\right) \\
& \times\left(\left(h_{2} h_{4}-h_{7}-h_{10}\right)-\lambda_{z}^{[1]}\right)\left(\left(h_{12}+e h_{2} h_{5}-q E\right)-\lambda_{p}^{[1]}\right)
\end{aligned}
$$

from which, we obtain that:

$\lambda_{y}^{[1]}=h_{2} h_{3}-h_{6}-h_{8}, \lambda_{z}^{[1]}=h_{2} h_{4}-h_{7}-h_{10}$,

$\lambda_{x}^{[1]}=-h_{1}$ and $\lambda_{p}^{[1]}=h_{12}+e h_{2} h_{5}-q E$

Here $\lambda_{x}^{[1]}, \lambda_{y}^{[1]}, \lambda_{z}^{[1]}$ and $\lambda_{p}^{[1]}$ denote to the eigenvalues in the $x$-direction, $y$-direction, $z$-direction and $p$-direction, respectively. So, it is easy to verify that, all the eigenvalues have negative real parts if and only if the condition (2) holds. Therefore, the equilibrium point $E_{1}$ is locally asymptotically stable in $\Re_{+}^{4}$.

Similarly, the equilibrium point $E_{2}$ is locally asymptotically stable if and only if $p_{2}>\frac{h_{1}}{h_{5}} \cdot E_{3}$ is locally asymptotically stable if and only if $h_{12}+e h_{5} x_{3}+e h_{9} y_{3}<q$ Eand $x_{3}<\frac{\left(h_{7}+h_{10}\right)}{h_{4}} \cdot E_{4}$ is locally asymptotically stable if and only if $2 x_{4}+z_{4}<h_{2} ; h_{12}+e h_{5} x_{4}+e h_{11} z_{4}<q E$ $x_{4}<\frac{\left(h_{6}+h_{8}\right)}{h_{3}}$ and $h_{1}<\frac{h_{2} h_{4} z_{4}}{\left(h_{2}-2 x_{4}-z_{4}\right)}$. $E_{5}$ is locally asymptotically stable if and only if $x_{5}<\min \left\{\frac{\left(h_{6}+h_{8}+h_{5} p_{5}\right)}{h_{3}}, \frac{\left(h_{7}+h_{10}+h_{11} p_{5}\right)}{h_{4}}\right\}$.

\section{Theorem (3):}

If the following conditions hold $h_{3} x_{6}<h_{6}+h_{8}+h_{9} p_{6}$

$$
\begin{aligned}
& h_{11} h_{13}<\min \left\{\frac{h_{4} h_{12}}{e h_{5}} ; \frac{h_{5} h_{12} p_{6}}{h_{4} z_{6}}\right\} \\
& h_{1} h_{2}<2 h_{1} x_{6}+\left(h_{2} h_{4}+h_{1}\right) z_{6}+h_{2} h_{5} p_{6} \\
& h_{2} h_{7}<\left(h_{2} h_{4}+h_{1}\right) x_{6}
\end{aligned}
$$

then $E_{6}$ is a locally asymptotically stable. 


\section{Proof:}

The Jacobian matrix of the system (1) at $E_{6}$ is given by:

$J_{6}=\left(\begin{array}{cccc}\beta_{11}^{[6]} & \beta_{12}^{[6]} & \beta_{13}^{[6]} & \beta_{14}^{[6]} \\ 0 & \beta_{22}^{[6]} & 0 & 0 \\ \beta_{31}^{[6]} & 0 & 0 & \beta_{34}^{[6]} \\ \beta_{41}^{[6]} & \beta_{42}^{[6]} & \beta_{43}^{[6]} & \beta_{44}^{[6]}\end{array}\right)$

where:

$\beta_{11}^{[6]}=h_{1}\left(1-\frac{2}{h_{2}} x_{6}\right)-z_{6}\left(h_{4}+\frac{h_{1}}{h_{2}}\right)-h_{5} p_{6}$;

$\beta_{12}^{[6]}=h_{6}-\left(\frac{h_{1}}{h_{2}}+h_{3}\right) x_{6} ; \beta_{13}^{[6]}=h_{7}-\left(\frac{h_{1}}{h_{2}}+h_{4}\right) x_{6}$;

$\beta_{14}^{[6]}=-h_{5} x_{6} ; \beta_{22}^{[6]}=h_{3} x_{6}-h_{6}-h_{8}-h_{9} p_{6} ;$

$\beta_{31}^{[6]}=h_{4} z_{6} ; \beta_{34}^{[6]}=-h_{11} z_{6} ; \beta_{41}^{[6]}=e h_{4} p_{6} ;$

$\beta_{42}^{[6]}=e h_{9} p_{6} ; \beta_{43}^{[6]}=e h_{11} p_{6} ; \beta_{44}^{[6]}=\frac{-h_{12}}{h_{13}} p_{6}$

So, the characteristic equation of $J_{6}$ can be written by

$$
\left.\left(\lambda_{y}^{[6]}-\beta_{22}^{[6]}\right)\left(\lambda^{[6]}\right)^{3}+F_{1}\left(\lambda^{[6]}\right)^{2}+F_{2}\left(\lambda^{[6]}\right)+F_{3}\right\rfloor=0
$$

with

$$
\begin{aligned}
F_{1} & =-\left(\beta_{11}^{[6]}+\beta_{44}^{[6]}\right) ; \\
F_{2} & =\beta_{11}^{[6]} \beta_{44}^{[6]}-\beta_{14}^{[6]} \beta_{41}^{[6]}-\beta_{13}^{[6]} \beta_{31}^{[6]}-\beta_{43}^{[6]} \beta_{34}^{[6]} ; \\
F_{3} & =\beta_{43}^{[6]} \beta_{34}^{[6]} \beta_{11}^{[6]}+\beta_{13}^{[6]} \beta_{31}^{[6]} \beta_{44}^{[6]}-\beta_{14}^{[6]} \beta_{43}^{[6]} \beta_{31}^{[6]} \\
& -\beta_{13}^{[6]} \beta_{34}^{[6]} \beta_{41}^{[6]}
\end{aligned}
$$

Here $\lambda_{y}^{[6]}$ denote to the eigenvalues in the $y$-direction. The Routh-Hurwitz conditions require $F_{1}>0 ; F_{3}>0$ and $\Delta=F_{1} F_{2}-F_{3}>0$,

which follows from condition (3b) and in addition the negativity of the other eigenvalues, namely condition (3a). So, according to Routh-Hurwitz criterion $E_{6}$ is locally asymptotically stable.

\section{Theorem (4):}

If the following conditions hold $h_{4} x_{7}<h_{7}+h_{10}+h_{11} p_{7}$

$h_{9} h_{13}<\min \left\{h_{3} h_{12}\left(e h_{5}\right)^{-1} ; h_{5} h_{12} p_{7}\left(h_{3} z_{7}\right)^{-1}\right\}$

$h_{1} h_{2}<2 h_{1} x_{7}+\left(h_{2} h_{3}+h_{1}\right) y_{7}+h_{2} h_{5} p_{7}$ $h_{2} h_{6}<\left(h_{2} h_{3}+h_{1}\right) x_{7}$

then $E_{7}$ is a locally asymptotically stable.

\section{Proof:}

Due to the form of eigenvalues of $J_{7}$, the proof is follows directly as that given in theorem(3).

\section{Theorem (5):}

The equilibrium point $E_{8}$ is locally asymptotically stable in the subregion in $\mathfrak{R}_{+}^{4}$ if and only if:

$x+y+z<h_{2} ; p<h_{13} ;$

$x<\min \left\{\frac{\left(h_{6} y+h_{7} z\right)}{\left(h_{3} y_{8}+h_{4} z_{8}\right)}, x_{8}\right\}$

$\left.e h_{5} x_{8}+e h_{9} y_{8}+e h_{11} z_{8}+h_{12}\left(1-\frac{p}{h_{13}}\right)<q E\right)$

\section{Proof:}

The Jacobian matrix of the system (1) at $E_{8}$ is given by:

$J_{8}=\left(\begin{array}{cccc}\beta_{11}^{[8]} & \beta_{12}^{[8]} & \beta_{13}^{[8]} & \beta_{14}^{[8]} \\ \beta_{21}^{[8]} & 0 & 0 & \beta_{24}^{[8]} \\ \beta_{31}^{[8]} & 0 & 0 & \beta_{34}^{[8]} \\ 0 & 0 & 0 & \beta_{44}^{[8]}\end{array}\right)$ where:

$\beta_{11}^{[8]}=h_{1}\left(1-\frac{2}{h_{2}} x_{8}\right)-y_{8}\left(h_{3}+\frac{h_{1}}{h_{2}}\right)-z_{8}\left(h_{4}+\frac{h_{1}}{h_{2}}\right) ;$

$\beta_{12}^{[8]}=-\left(\frac{h_{1}}{h_{2}} x_{8}+h_{8}\right) ; \beta_{13}^{[8]}=-\left(\frac{h_{1}}{h_{2}} x_{8}+h_{10}\right) ;$

$\beta_{14}^{[8]}=-h_{5} x_{8} ; \beta_{21}^{[8]}=h_{3} y_{8} ; \beta_{24}^{[8]}=-h_{9} y_{8} ; \beta_{31}^{[8]}=h_{4} z_{8} ;$

$\beta_{34}^{[8]}=-h_{11} z_{8} ; \beta_{44}^{[8]}=h_{12}+e\left(h_{5} x_{8}+h_{9} y_{8}+h_{11} z_{8}\right)-q E$

So, the characteristic equation of $J_{8}$ can be written by

$\left.\lambda^{[8]}\left(\lambda_{p}^{[8]}-\beta_{44}^{[8]}\right)\left(\lambda^{[8]}\right)^{2}-\beta_{11}^{[8]} \lambda^{[8]}-\left(\beta_{13}^{[8]} \beta_{31}^{[8]}+\beta_{12}^{[8]} \beta_{21}^{[8]}\right)\right)=0$

Since $\lambda^{[8]}=0$ that mean $E_{8}$ is non-hyperbolic equilibrium point, then consider the function

$$
\begin{aligned}
V^{[8]} & =\left(x-x_{8}-x_{8} \ln \frac{x}{x_{8}}\right)+\left(y-y_{8}-y_{8} \ln \frac{y}{y_{8}}\right) \\
& +\left(z-z_{8}-z_{8} \ln \frac{z}{z_{8}}\right)+\frac{p}{e}
\end{aligned}
$$

Clearly, $V^{[8]}: \mathfrak{R}_{+}^{4} \rightarrow \mathfrak{R}$ and $V^{[8]}\left(E_{8}\right)=0$ with $V^{[8]}(E) \neq 0 \quad \forall E \neq E_{8}, E \in \mathfrak{R}_{+}^{4}$. Hence it is positive definite function in $\mathfrak{R}_{+}^{4}$. Also, the derivative of $V^{[8]}$ with respect to the time $t$ is given as follows.

$$
\begin{aligned}
& \frac{d V^{[8]}}{d t}=\left[\begin{array}{c}
\left.h_{1}\left(1-\frac{x+y+z}{h_{2}}\right)+\frac{\left(h_{6} y+h_{7} z\right)}{x}\right]\left(x-x_{8}\right) \\
-h_{3} y_{8}-h_{4} z_{8}
\end{array}\right] \\
& +\frac{p}{e}\left[e h_{5} x_{8}+e h_{9} y_{8}+e h_{11} z_{8}+h_{12}\left(1-\frac{p}{h_{13}}\right)-q E\right]
\end{aligned}
$$


In addition condition(5) guarantee that $\frac{d V^{[8]}}{d t}<0$ on subregion of $\Re_{+}^{4}$, then $V^{[8]}$ is a Lyapunov function on that subregion. Therefore $E_{8}$ is a locally asymptotically stable but not globally.

\section{Theorem (6):}

The coexistence equilibrium point $E_{9}$ is locally asymptotically stable in $\Re_{+}^{4}$ if and only if

$$
\begin{aligned}
& \frac{h_{4} h_{9} \beta_{11}^{[9]}}{h_{3} \beta_{44}^{[9]}}<h_{11}<\min \left\{\begin{array}{c}
\frac{h_{9}\left(h_{2} h_{10}+h_{1} x_{9}\right)}{\left(h_{2} h_{8}+h_{1} x_{9}\right)}\left(1, \frac{\beta_{44}^{[9]}}{\beta_{11}^{[9]}}\right), \\
\frac{h_{4} h_{9}}{h_{3}}\left(1, \frac{\beta_{44}^{[9]}}{\beta_{11}^{[9]}}\right)
\end{array}\right\} \\
& h_{1}<\frac{2 h_{1}}{h_{2}} x_{9}+y_{9}\left(h_{3}+\frac{h_{1}}{h_{2}}\right)+z_{9}\left(h_{4}+\frac{h_{1}}{h_{2}}\right)+h_{5} p_{9} \text {; } \\
& e h_{5} h_{13}<\min \left\{\frac{h_{4} h_{12}}{h_{11}}, \frac{h_{3} h_{12}}{h_{9}}\right\} \\
& x_{9}<\min \left\{\frac{h_{9} h_{12} p_{9}}{h_{3} h_{5} h_{13}}, \frac{h_{11} h_{12} p_{9}}{h_{4} h_{5} h_{13}}\right\}
\end{aligned}
$$

\section{Proof:}

The Jacobian matrix of the system (1) at $E_{9}$ is given by:

$$
\begin{aligned}
& J_{9}=\left(\begin{array}{cccc}
\beta_{11}^{[9]} & \beta_{12}^{[9]} & \beta_{13}^{[9]} & \beta_{14}^{[9]} \\
\beta_{21}^{[9]} & 0 & 0 & \beta_{24}^{[9]} \\
\beta_{31}^{[9]} & 0 & 0 & \beta_{34}^{[9]} \\
\beta_{41}^{[9]} & \beta_{42}^{[9]} & \beta_{43}^{[9]} & \beta_{44}^{[9]}
\end{array}\right) \text { Where: } \\
& \beta_{11}^{[9]}=h_{1}\left(1-\frac{2 x_{9}}{h_{2}}\right)-y_{9}\left(h_{3}+\frac{h_{1}}{h_{2}}\right)-z_{9}\left(h_{4}+\frac{h_{1}}{h_{2}}\right)-h_{5} p_{9} ; \\
& \beta_{12}^{[9]}=-\left(h_{8}+h_{9} p_{9}+\frac{h_{1}}{h_{2}} x_{9}\right) ; \beta_{14}^{[9]}=-h_{5} x_{9} ; \\
& \beta_{13}^{[9]}=-\left(h_{10}+h_{11} p_{9}+\frac{h_{1}}{h_{2}} x_{9}\right) ; \beta_{21}^{[9]}=h_{3} y_{9} ; \beta_{24}^{[9]}=-h_{9} y_{9} ; \\
& \beta_{31}^{[9]}=h_{4} z_{9} ; \beta_{34}^{[9]}=-h_{11} z_{9} ; \beta_{41}^{[9]}=e h_{5} p_{9} ; \beta_{42}^{[9]}=e h_{9} p_{9} ; \\
& \beta_{43}^{[9]}=e h_{11} p_{9} ; \beta_{44}^{[9]}=\frac{-h_{12}}{h_{13}} p_{9}
\end{aligned}
$$

So, the characteristic equation of $J_{9}$ can be written by

$$
\left\lfloor\left(\lambda^{[9]}\right)^{4}+F_{1}\left(\lambda^{[9]}\right)^{3}+F_{2}\left(\lambda^{[9]}\right)^{2}+F_{3}\left(\lambda^{[9]}\right)+F_{4}\right\rfloor=0
$$

$$
\text { where: }
$$

$$
\begin{aligned}
F_{1}= & -\left(\beta_{11}^{[9]}+\beta_{44}^{[9]}\right) ; F_{2}=\beta_{11}^{[9]} \beta_{44}^{[9]}-\beta_{13}^{[9]} \beta_{31}^{[9]} \\
& -\beta_{43}^{[9]} \beta_{34}^{[9]}-\beta_{14}^{[9]} \beta_{41}^{[9]}-\beta_{12}^{[9]} \beta_{21}^{[9]}-\beta_{24}^{[9]} \beta_{42}^{[9]}
\end{aligned}
$$

$$
\begin{aligned}
F_{3} & =\beta_{13}^{[9]} \beta_{31}^{[9]} \beta_{44}^{[9]}-\beta_{13}^{[9]} \beta_{34}^{[9]} \beta_{41}^{[9]}-\beta_{14}^{[9]} \beta_{43}^{[9]} \beta_{31}^{[9]} \\
& +\beta_{43}^{[9]} \beta_{34}^{[9]} \beta_{11}^{[9]}+\beta_{12}^{[9]} \beta_{21}^{[9]} \beta_{44}^{[9]}-\beta_{14}^{[9]} \beta_{42}^{[9]} \beta_{21}^{[9]} \\
& -\beta_{12}^{[9]} \beta_{24}^{[9]} \beta_{41}^{[9]}+\beta_{24}^{[9]} \beta_{42}^{[9]} \beta_{11}^{[9]} \\
F_{4} & =\beta_{12}^{[9]} \beta_{21}^{[9]} \beta_{34}^{[9]} \beta_{43}^{[9]}-\beta_{13}^{[9]} \beta_{34}^{[9]} \beta_{42}^{[9]} \beta_{21}^{[9]} \\
& +\beta_{13}^{[9]} \beta_{31}^{[9]} \beta_{24}^{[9]} \beta_{42}^{[9]}-\beta_{12}^{[9]} \beta_{24}^{[9]} \beta_{43}^{[9]} \beta_{31}^{[9]}
\end{aligned}
$$

Hence, the Routh-Hurwitz conditions require $F_{1}>0 ; F_{3}>0 ; F_{4}>0 ; \Delta=F_{1} F_{2}-F_{3}>0$ and $\left(F_{1} F_{2}-F_{3}\right) F_{3}-\left(F_{1}\right)^{2} F_{4}>0$, which follows from condition (6). So, according to Routh-Hurwitz criterion $E_{9}$ is locally asymptotically stable.

\section{Numerical Simulations}

The system (1) is solved numerically for different sets of parameters with different initial conditions, and then the time series for the trajectories of system (1) are drawn to confirm our obtained analytical results. Note that we will use the cont. line (-) for $x$, dash line(- -) for $y$, dot line(::) for $z$ and dash-dot line(-.-) for $p$ in the all of the following figures. Now, for the following parameters:

$$
\begin{aligned}
& h_{1}=0.02, h_{2}=0.4, h_{3}=0.9, h_{4}=0.9, h_{5}=0.8, \\
& h_{6}=0.05, h_{7}=0.05, h_{8}=0.01, h_{9}=0.29, \\
& h_{10}=0.01, h_{11}=0.31, h_{12}=0.03, h_{13}=0.6, \\
& q=0.2, e=0.8, E=0.4
\end{aligned}
$$

The time series of the trajectories of system (1) are drown in Fig.(1).

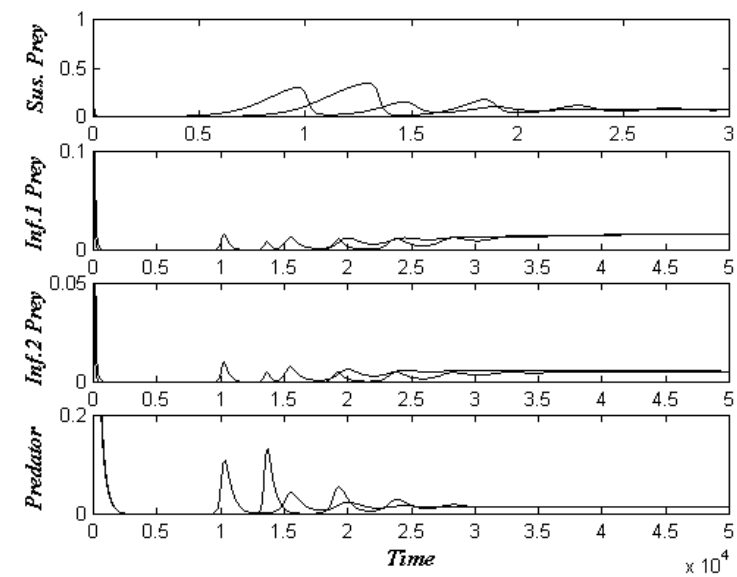

Fig.(1) The time series for the trajectories of system(1) starting from different initial points.

According to the above figure, system(1) approaches asymptotically to the stable coexistence equilibrium point $E_{9}=(0.0712,0.0165,0.0051,0.0138) \quad$ starting from different initial points $(0.5,0.5,0.5,0.5)$, $(0.75,0.75,0.75,0.75),(1,1,1,1)$. 
In order to investigate the effect of infection rate, due to existence of first disease, (i.e. parameter $h_{3}$ ) on the dynamics of system (1) in case of existence of $E_{9}$, the system is solved numerically for different values of $h_{3}$ with $h_{3}=0.8,0.91$ while the rest of parameters as given in Eq. (7) and then the trajectories of system (1) are drown in the Fig.(2.a)-(2.b).

(a)

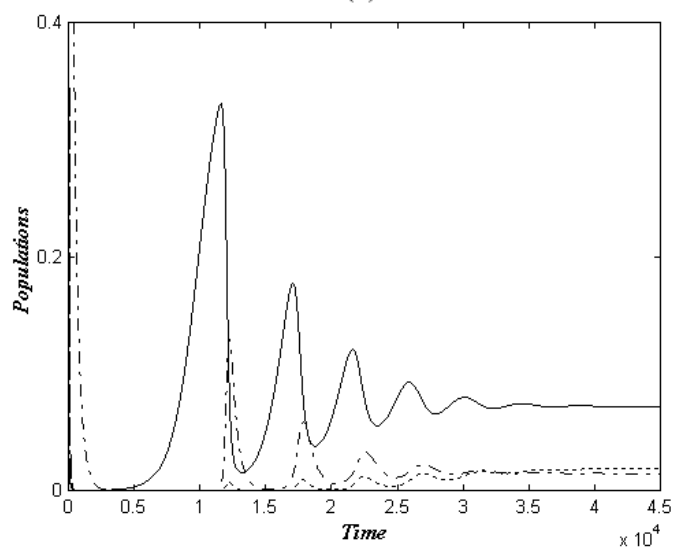

(b)

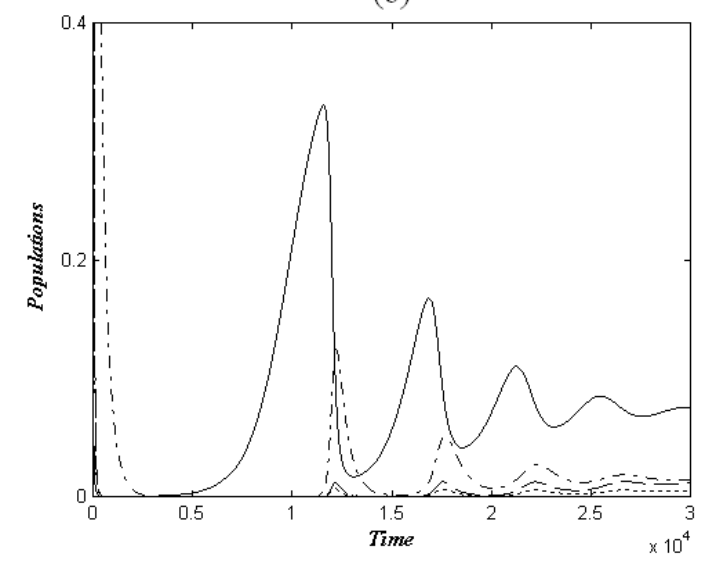

Fig.(2) Time series of trajectories for data given in Eq.(7) with different values of $h_{3}$ which shows that the trajectories

approaches asymptotically to the stable point:(a) $E_{6}$ for $h_{3}=0.8$ (b) $E_{9}$ for $h_{3}=0.91$.

According to the above results, it is observed that the trajectory of system (1) approaches to stable point $E_{6}=(0.0716,0,0.0195,0.0144)$ for $h_{3}<0.88$ as shown in the typical Fig. (2.a), while it is approaches to asymptotically stable point $E_{9}=(0.0712,00165,0.005,0.0138)$ for $0.88 \leq h_{3}$ as shown in the typical Fig.(2.b). Similar observations have been obtained for increasing the recovery rate $h_{6}$ on the dynamical behavior of system (1).
Now the effect of infection rate, due to existence of second disease, on the dynamical behavior of the system (1) is studied numerically for parameters values given in Eq.(7) with $h_{4}=0.8,0.92$ and the time series are drawn in Fig.(3.a)-(3.b).

(a)

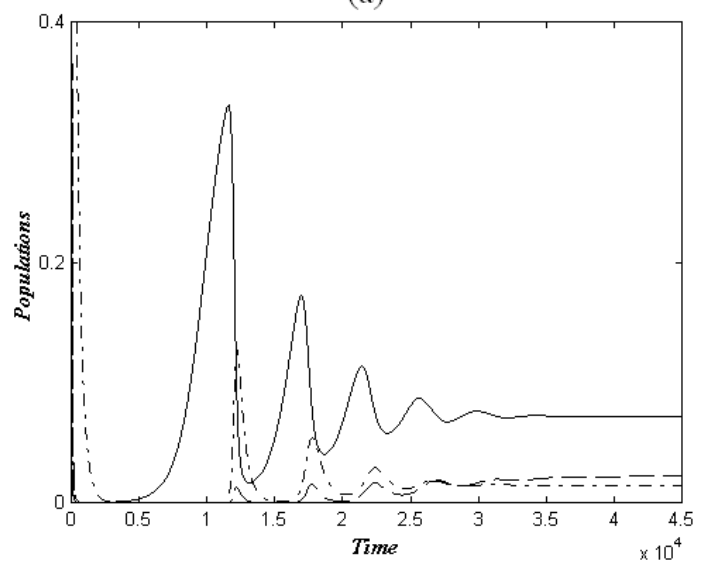

(b)

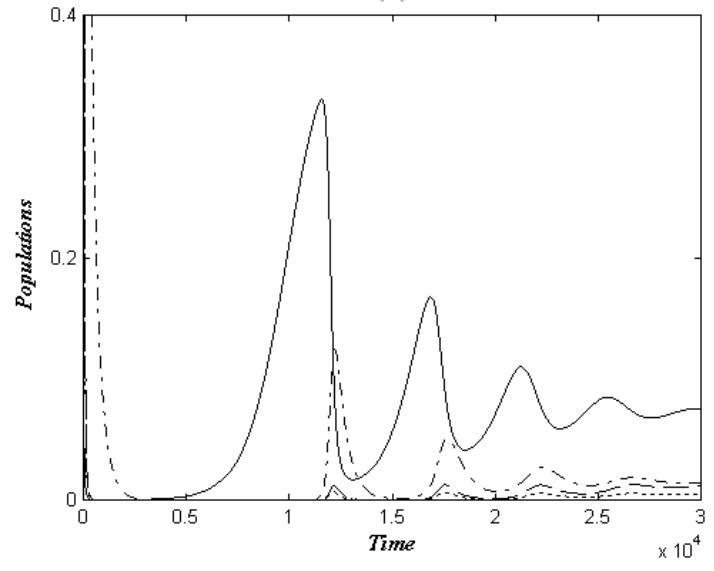

Fig.(3) Time series of the trajectories for data given in Eq.(7) with different values of $h_{4}$ which shows that the trajectories approaches asymptotically to the stable point: (a) $E_{7}$ for

$$
h_{4}=0.8 \text { (b) } E_{9} \text { for } h_{4}=0.92 \text {. }
$$

Clearly, from the above figures, it is observed that decreasing the value of the infection rate causes decreasing in $z$ and then the system (1) approaches to stable point $E_{7}=(0.0711,0.0223,0,0.0137)$ in $x y p-$ plane that is means disappearing of the second disease. Similar observations have been obtained for increasing the recovery rate $h_{7}$ on the dynamical behavior of system (1).

Finally, we will investigate the effect of varying harvest rate $q E$, which is the catch ability co-efficient of the predator, on the dynamics of system (1). again the system(1) 
solved numerically for the values $q E$ while the rest of parameters as given in Eq.(7) and then the trajectories of system(1) are drawn in the Figs.(4.a)-(4.c) for $q E=0.03,0.05,0.1$.

(a)

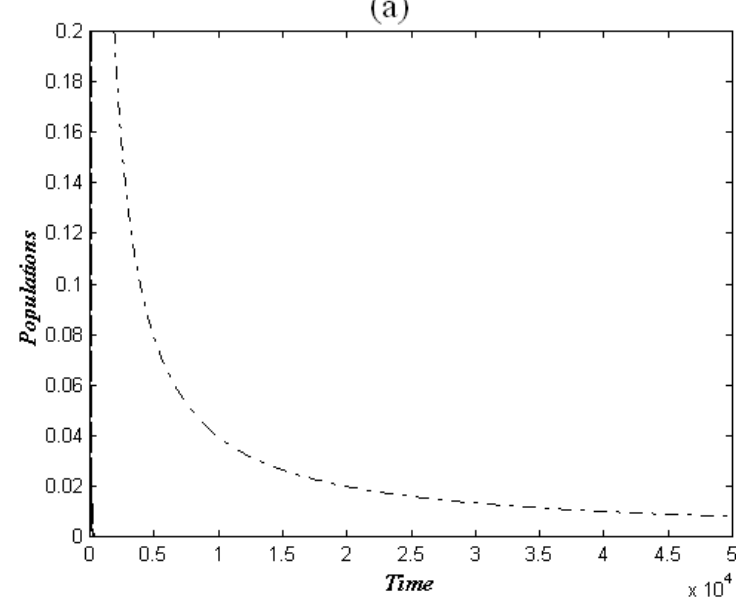

(b)

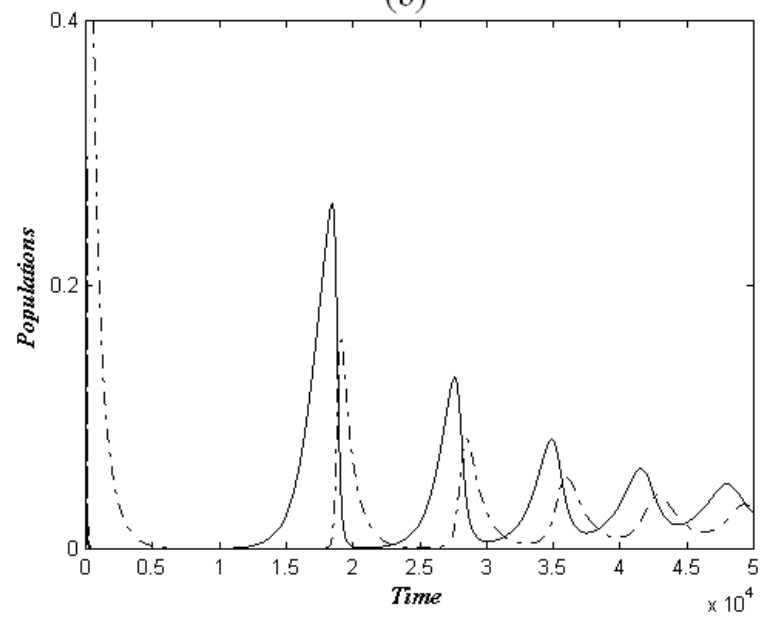

(c)

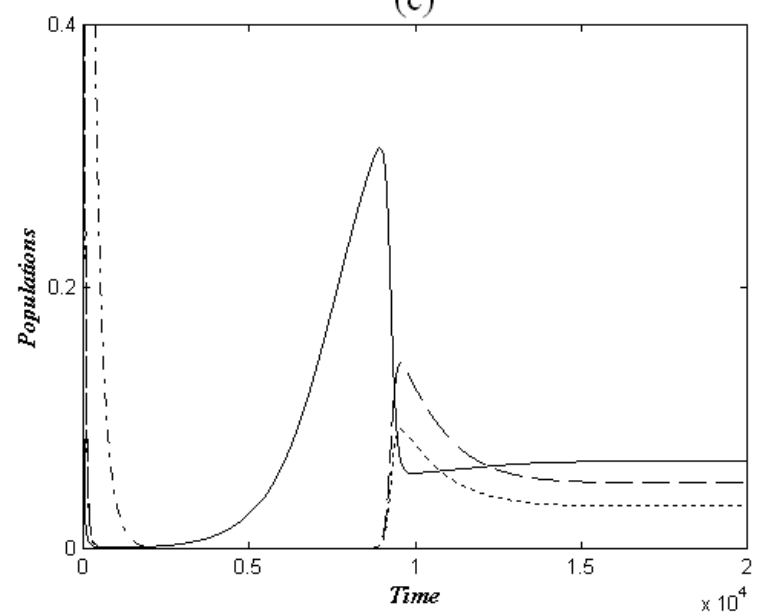

Fig.(4) Time series of the trajectories of system(1) for data given in Eq.(7) with different values of $q E$ which shows that the

trajectories approaches asymptotically to the stable point: (a) $E_{2}$ for $q E=0.03$ (b) $E_{5}$ for $q E=0.05(c) E_{8}$ for $q E=0.1$.
Obviously, from the above figures, as $q E$ increases causes decreasing in the values of $p$ species while the value of $x, y, z$ species are increasing and the trajectory of system (1) approaches to the equilibrium point $E_{2}=(0,0,0,0.08)$ as shown in Fig.(4.a). However the trajectory of system (1) approaches asymptotically to stable point $E_{5}=(0.0264,00,0.031)$ as shown in Fig.(4.b), while it is approaches to $E_{8}=(0.0667,0051,0.0327,0)$ as shown in Fig.(4.c).

\section{Conclusions and Discussion}

In this paper, an eco-epidemiological model has been proposed and analysed. In order to study the effect of two infection diseases and harvesting on the dynamical behavior of the prey-predator system, the dynamical behavior of system (1) has been investigated locally as well as globally. Now, we shall discuss the effects of changing the parameters on the dynamical behavior of system (1) according to the numerical results:

1. For the effect of the varying values of $h_{3}$, keeping other parameters fixed as in Eq.(7), when the infection rate by first disease in the rang $0.1 \leq h_{3}<0.87$ then the trajectories of system (1) approaches to free first disease equilibrium point $E_{6}$, and if infection rate increasing $h_{3} \geq 0.88$ that implies the first disease appears and the trajectories of system (1) approaches to coexistence equilibrium points $E_{9}$.similar effect have been obtained for increasing the recovery rate $h_{6}$ on the dynamical behavior of system (1).

2. If the infection rate by second disease in $\left(0.1 \leq h_{4}<0.8\right)$ the value of $z$ disappear and then the system (1) approaches to stable point $E_{7}$, for $h_{4} \geq 0.8$, the trajectory of system (1) approaches to $E_{9}$. Similar observations have been obtained for increasing the recovery rate $h_{7}$ on the dynamical behavior of system (1).

3. For small value of harvest rate say $(0.01<q E \leq 0.03)$ the trajectory of system (1) approaches to $E_{2}$. As the harvest rate increases $0.05<q E<0.08$ the trajectory of 
system (1) approaches to $E_{5}$. While for $0.08 \leq q E<0.1$, the trajectory of system (1) approaches to $E_{9}$. Finally, for $q E \geq 0.1$, the predator evanescent.

\section{References}

[1] Anderson R. M. and May R. M., "The invasion, persistence, and spread of infectious diseases within animal and plant communities", Philos. Trans. R. Soc. Lond. B., 314, 533, 1986.

[2] Andreasen V. and Sasaki A., "Shaping the phylogenetic tree of influenza by crossimmunity", Theoretical population biology, 70, 164-173, 2006.

[3] Arino O., Abdllaoui A., Mikram J., and Chattopadhyay J., "Infection in prey population may act as a biological control in ratio-dependent predator-prey models", Nonlinearity, 17, 1101-1116, 2004.

[4] Bakare E. A., Adekunle Y. and Nwagwo A., "Mathematical analysis of the control of the spread of infectious disease in a prey-predator ecosystem", International Journal of Computer \& Organization Trends, 2(1), 27-32, 2012.

[5] Chattopadhyay J. and Arino O., "A predator-prey model with disease in the prey", Nonlinear Analysis, 36, 747-766, 1999.

[6] Das K. P., "A Mathematical study of a predator-prey dynamics with disease in predator", ISRN Applied Mathematics, 2011, 1-16, 2011.

[7] Haque M. and Venturino E., "An ecoepidemiological model with disease in predator: the ratio-dependent case", Mathematical Methods in the Applied Sciences, 30, 1791-1809, 2007.

[8] Haque M. and Venturino E., "Increase of the prey may decrease the healthy predator population in presence of disease in the predator", HERMIS, 7, 38-59, 2006.

[9] Haque M. and Venturino E., "The effect of communicable disease in Leslie-Gower predator-prey model", Journal of Biological System, 163, 425-444, 2008.

[10] Haque M., "A predator-prey model with disease in the predator species only", Nonlinear Analysis. RWA, 11(4), 22242236, 2010.
[11] Haque M., Sarwardi S., Preston S. and Venturino E., "Effect of delay in a LotkaVolterra type predator-prey model with a transmissible disease in the predator species", Mathematical Biosciences, 234(1), 47-57, 2011.

[12] Brauer F. and Soudack A. C., "Stability regions and transition phenomena for harvested predator-prey systems", J. Math. Biol., 7, 319-337, 1979.

[13] Brauer F. and Soudack A. C., "Stability regions in predator-prey systems with constant-rate prey harvesting", J. Math. Biol., 8, 55-71, 1979.

[14] Dai G. and Tang M., "Coexistence region and global dynamics of a harvested predator-prey system", SIAM J. Appl. Math., 58, 193-210, 1998.

[15] Myerscough M. R., Gray B. F., Hogarth W. L., Norbury J., "An analysis of an ordinary differential equation model for a two-species predator-prey system with harvesting and stocking", J. Math. Biol., 30, 389-411, 1992.

[16] Xiao D. and Ruan S., "Bogdanov-Takens bifurcations in predator-prey systems with constant rate harvesting", Fields Institute Communications, 21, 493506,1999.

[17] Rasha Majeed Yaseen, “A Predator-Prey Model with Transition Two Infectious Diseases in Prey Population and Harvesting of The Predator Population", Journal of Mathematical Theory and Modeling, 4(10), 1-27, 2014.

[18] Fabio Roman, Federica Rossotto and Ezio Venturino, "Ecoepidemics with two strains: diseased predators", WSEAS Transactions on Biology and Biomedicine, 8(3), 73-85, 2011.

[19] Elisa Elena, Maria Grammauro and Ezio Venturino, "Predator's alternative food sources do not support ecoepidemics with two strains-diseased prey", Network Biology, 3(1), 29-44, 2013.

[20] Hirsch M. W. and Smale S., "Differential Equation, Dynamical System, and Linear Algebra", New York, Academic Press, 1974. 
[21] Haberman R., "Mathematical Models", New Jersey, Prentice-Hall Inc., 1977.

[22] Earn D. J., Dushoff D. J. and Levin S. A., "Ecology and evolution of the flu.", Trends in Ecology and Evolution, 17, 334-340,2002.

[23] Ferguson N.M., Galvani A.P. and Bush R.M., "Ecological and immunological determinants of influenza evolution", Nature, 422, 428-433, 2003.

[24] Brauer F., Wu J. and Driessche P., "Mathematical epidemiology", Berlin Heidelberg, Springer-Verlag, 2008.

[25] Chasnov J. R., "Mathematical Biology", Jeffrey Robert Chasnov, USA, 2012.

[26] Murray J. D., "Mathematical biology an introduction", third edition, Berlin Heidelberg, Springer-Verlag, 2002.

[27] Li Li and Han B., "A dynamical system method for solving nonlinear ill-posed problems", Applied Mathematics and computation, 197(1),399-406, 2008, المكتبة الافتر اضية.

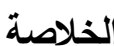

في هذا البحث، اقترحنا ودرسنا نموذج بيئي -وبائي مع

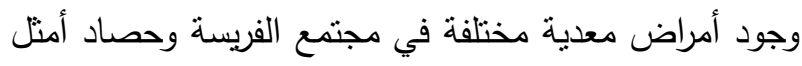

في مجتمع المفترس. أستخدمنا صيغة خطية لوصف استجابة

وظيفي، أيجاد الثروط الكافية التي تضمن وجود كل نقاط

التوازن المكنة في هذا النظام، قمنا بدراسة تحليل الاستقرارية

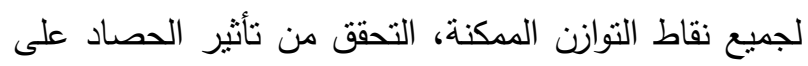

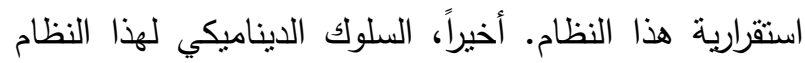

تمت مناقتته بأستخدام المحاكاة العددية. 